\title{
RECTIFIABILITY OF SELF-CONTRACTED CURVES IN THE EUCLIDEAN SPACE AND APPLICATIONS
}

\author{
A. DANIILIDIS, G. DAVID, E. DURAND-CARTAGENA, A. LEMENANT
}

\begin{abstract}
It is hereby established that, in Euclidean spaces of finite dimension, bounded selfcontracted curves have finite length. This extends the main result of 6 concerning continuous planar self-contracted curves to any dimension, and dispenses entirely with the continuity requirement. The proof borrows heavily from a geometric idea of [13] employed for the study of regular enough curves, and can be seen as a nonsmooth adaptation of the latter, albeit a nontrivial one. Applications to continuous and discrete dynamical systems are discussed: continuous self-contracted curves appear as generalized solutions of nonsmooth convex foliation systems, recovering a hidden regularity after reparameterization, as consequence of our main result. In the discrete case, proximal sequences (obtained through implicit discretization of a gradient system) give rise to polygonal self-contracted curves. This yields a straightforward proof for the convergence of the exact proximal algorithm, under any choice of parameters.
\end{abstract}

\section{Contents}

1. Introduction 2

1.1. Motivation and state-of-the-art 2

1.2. Contributions of this work 3

1.3. Acknowledgements 4

2. Notation and preliminaries 4

2.1. Self-expanded curves 5

2.2. Self-contracted versus self-expanded curves 6

3. Rectifiability of self-contracted curves

3.1. Proof of the main result 7

3.2. Arc-length reparameterization 14

4. Applications 15

4.1. Orbits of convex foliations

4.2. Polygonal approximations of smooth strongly self-contracted curves

4.3. Convergence of the proximal algorithm 21

References

2000 Mathematics Subject Classification. Primary 53A04 ; Secondary 37N40, 49J52, 49J53, 52A10, 65K10.

Key words and phrases. Self-contracted curve, rectifiable curve, convex foliation, secant, self-expanded curve, proximal algorithm. 


\section{INTRODUCTION}

1.1. Motivation and state-of-the-art. Self-contracted curves were introduced in [6, Definition 1.2.] to provide a unified framework for the study of convex and quasiconvex gradient dynamical systems. Given a possibly unbounded interval $I$ of $\mathbb{R}$, a map $\gamma: I \rightarrow \mathbb{R}^{n}$ is called self-contracted curve, if for every $[a, b] \subset I$, the real-valued function

$$
t \in[a, b] \mapsto d(\gamma(t), \gamma(b))
$$

is non-increasing. This notion is purely metric and does not require any prior smoothness/continuity assumption on $\gamma$.

So far, self-contracted curves are considered in a Euclidean framework. In particular, given a smooth function $f: \mathbb{R}^{n} \rightarrow \mathbb{R}$, any solution $\gamma$ of the gradient system

$$
\left\{\begin{array}{l}
\gamma^{\prime}(t)=-\nabla f(\gamma(t)) \quad t>0 \\
\gamma(0)=x_{0} \in \mathbb{R}^{n}
\end{array}\right.
$$

is a (smooth) self-contracted curve, provided that $f$ is quasiconvex, that is, its sublevel sets

$$
[f \leq \beta]:=\left\{x \in \mathbb{R}^{n}: f(x) \leq \beta\right\} \quad(\beta \in \mathbb{R})
$$

are convex subsets of $\mathbb{R}^{n}[6$, Proposition 6.2]. Self-contracted curves also appear in subgradient systems, defined by a (nonsmooth) convex function $f$, see [6, Proposition 6.4]. In this case, the first equation in (1.1) becomes the following differential inclusion

$$
\gamma^{\prime}(t) \in-\partial f(\gamma(t)) \text { a.e., }
$$

where a.e. stands for "almost everywhere" and the solutions are absolutely continuous curves (see [2] for a general theory).

A central question of the asymptotic theory of a general gradient dynamical system of the form (1.1) is whether or not bounded orbits are of finite length, which if true, yields in particular their convergence. This property fails for $C^{\infty}$ smooth functions ([15, p. 12]), but holds for analytic gradient systems ([11]), or more generally, for systems defined by an o-minimal (tame) function ([9]). In these cases, a concrete estimation of the length of (sub)gradient curves is obtained in terms of a so-called Łojasiewicz type inequality, intrinsically linked to the potential $f$, see [1] for a survey, which also includes extensions of the theory to subgradient systems. Notice however that convex functions fail to satisfy such inequality, see [1, Section 4.3] for a counterexample.

In [13] a certain class of Lipschitz curves has been introduced (with no specific name) to capture the behaviour of orbits of quasiconvex potentials. Unlike self-contracted curves, this notion makes sense only in Euclidean spaces (it uses orthogonality) and requires the curve to be Lipschitz continuous. The main result of [13] asserts that the length of such curves is bounded by the mean width of their convex hull, a fortiori, by the mean width of any convex set containing the curve. Recently, the authors of [7] extended the result of [13] to 2-dimensional surfaces of constant curvature, naming these curves as $(\mathrm{G})$-orbits. We shall prefer to call these curves self-expanding, see Definition 2.2 below. The choice of this terminology will become clear in Section 2.2,

Formally the result, as announced in [13, IX], applies only under the prior requirement that the length of the curve is finite, since the proof is given for self-expanded curves parameterized by the arc-length parametrization in a compact interval. This restriction is removed in Section 2.1 via a simple continuity argument (see Corollary 2.4. As a result, the smooth orbits of (1.1) for a quasiconvex potential, as well as the absolutely continuous orbits of 1.2 for a convex one 
have finite length. In both cases the bound depends only on the diameter of the initial sublevel set.

The work [13] was unknown to the authors of [6], who tackled the same problem in terms of the aforementioned notion of self-contracted curve. The definition of self-contracted curve does not require any regularity neither on the space nor on the curve. In particular, such curves need not be continuous, and differentiability may a priori fail at each point. The main result of [6] shows that bounded continuous planar self-contracted curves have finite length ([․ Theorem 1.3]). This has been used to deduce that in $\mathbb{R}^{2}$, smooth orbits of quasiconvex systems (respectively, absolutely continuous orbits of nonsmooth convex systems) have finite length. As we saw before, this conclusion essentially derives from [13, IX] for any dimension (see comments above). Notice however that the main result of [6] cannot be deduced from [13], but can only be compared in retrospect, once rectifiability is established.

On the other hand, not completely surprisingly, Lipschitz continuous self-contracted curves and self-expanded curves turn out to be intimately related and can be obtained one from the other by means of an adequate reparameterization, reversing orientations (see Lemma 2.8). Recall however that both rectifiability and Lipschitz continuity of the curve are prior requirements for the definition of a self-expanded curve, while they are neither requirements nor obvious consequences of the definition of a self-contracted curve.

1.2. Contributions of this work. In this work we establish rectifiability of any self-contracted curve in $\mathbb{R}^{n}$, by extending the result of [6] to any dimension, and to possibly discontinuous curves. This is done by adapting the geometrical idea of [13] to the class of self-contracted curves. This nonsmooth adaptation is natural but quite involved. Nonsmooth variations of the mean width of the closed convex hull of the curve are again used to control the increase of its length, but no prior continuity on the parametrization is required and rectifiability is now part of the conclusions. Namely, setting $\Gamma=\gamma(I)$ (the image of the curve in $\mathbb{R}^{n}$ ) and denoting by $\ell(\gamma)$ its length, we establish the following result (see Theorem 3.3 ) :

- Every self-contracted curve is rectifiable and satisfies the relation $\ell(\gamma) \leq C \operatorname{diam}(\Gamma)$, where $C>0$ depends only on the dimension.

In case of continuous curves, the above result allows to consider a Lipschitz reparameterization defined by the arc-length, see details in Section 3.2. This leads to the following conclusion:

- If $\gamma$ is a continuous self-contracted curve and $\Gamma=\gamma(I)$ is bounded, then $\Gamma$ is also the image of some (Lipschitz) self-expanded curve.

In particular, the sets of all possible images of continuous self-contracted curves and of selfexpanded curves coincide. Still, the set of images of all self-contracted curves is much larger (its elements are not connected in general).

In the last two sections, two new applications of self-contracted curves are considered. In Section 4.1 we broaden the framework of dynamical systems to encompass nonsmooth convex foliation systems, with merely continuous generalized orbits. Limits of backward secants remedy the absence of differentiability, leading to a consistent notion of generalized solution in the sense of nonsmooth analysis (Definition 4.4). In Theorem 4.6 we show that these generalized solutions are self-contracted curves, thus of finite length; in view of the aforementioned result, they can also be obtained as "classical" solutions through an adequate Lipschitz reparameterization. On the other hand, $C^{1}$ smooth convex foliation orbits enjoy a stronger property, the so-called strong self-contractedness, see Definition 4.8 and Corollary 4.11. Concerning this latter class, 
we establish in Section 4.2 the following approximation result, with respect to the Hausdorff distance, see Proposition 4.13 .

- Every $C^{1}$-smooth strongly self-contracted curve is a limit of polygonal self-contracted curves.

Finally, in Section 4.3 we provide an elegant application of the notion of self-contracted curve in a different framework, that of discrete systems. In particular we establish the following result (Theorem 4.17) :

- Let $f$ be any convex function, bounded from below. Then the exact proximal algorithm gives rise to a self-contracted polygonal curve.

In view of our main result, we obtain a straightforward proof of the convergence of the proximal algorithm. The bound over the length of the polygonal curve yields a sharp estimation on the rate of convergence, which appears to be entirely new. Notice that the convergence result is independent of the choice of the parameters.

1.3. Acknowledgements. The first author acknowledges support of the grant MTM201129064-C01 (Spain) and thanks Jerome Bolte and Joel Benoist for useful discussions. The third author is partially supported by grant MTM2009-07848 (Spain). The second and fourth authors are partially supported by the ANR project GEOMETRYA (France). Part of this work has been realized during a research stay of the third author at the Université Paris Diderot (Paris 7) and Laboratory Jacques Louis Lions. The stay was supported by the program "Research in Paris" offered by the Ville de Paris (Mairie de Paris). This author thanks the host institution and Ville de Paris for its hospitality.

\section{NotATION AND PRELIMINARIES}

Let $\left(\mathbb{R}^{n}, d, \mathscr{L}^{n}\right)$ denote the $n$-dimensional Euclidean space endowed with the Euclidean distance $d(x, y)=\|x-y\|$, the scalar product $\langle\cdot, \cdot\rangle$, and the Lebesgue measure $\mathscr{L}^{n}$. We denote by $B(x, r)$ (respectively, $\bar{B}(x, r)$ ) the open (respectively, closed) ball of radius $r>0$ and center $x \in \mathbb{R}^{n}$. If $A$ is a nonempty subset of $\mathbb{R}^{n}$, we denote by $\sharp A$ its cardinality, by conv $(A)$ its convex hull and by $\operatorname{diam} A:=\sup \{d(x, y): x, y \in A\}$ its diameter. We also denote by $\operatorname{int}(A), \bar{A}$ and $\partial A$ the interior, the closure and respectively, the boundary of the set $A$.

Let now $K$ be a nonempty closed convex subset of $\mathbb{R}^{n}$ and $u_{0} \in K$. The normal cone $N_{K}\left(u_{0}\right)$ is defined as follows:

$$
N_{K}\left(u_{0}\right)=\left\{v \in \mathbb{R}^{n}:\left\langle v, u-u_{0}\right\rangle \leq 0, \forall u \in K\right\} .
$$

Notice that $N_{K}\left(u_{0}\right)$ is always a closed convex cone. Notice further that $u_{0} \in K$ is the projection onto $K$ of all elements of the form $u_{0}+t v$, where $t \geq 0$ and $v \in N_{K}\left(u_{0}\right)$.

The Hausdorff distance between two nonempty closed subsets $K_{1}, K_{2}$ of $\mathbb{R}^{n}$ is given by the formula

$$
d_{H}\left(K_{1}, K_{2}\right):=\inf \left\{\varepsilon>0: K_{2} \subset \bigcup_{z \in K_{1}} B(z, \varepsilon) \text { and } K_{1} \subset \bigcup_{z \in K_{2}} B(z, \varepsilon)\right\} .
$$

A sequence of closed sets $\left\{K_{j}\right\}_{j}$ in $\mathbb{R}^{n}$ is said to converge with respect to the Hausdorff distance to a closed set $K \subset \mathbb{R}^{n}$ if $d_{H}\left(K_{j}, K\right) \rightarrow 0$ as $j \rightarrow \infty$. 
Throughout the manuscript, $I$ will denote a possibly unbounded interval of $\mathbb{R}$. In this work, a usual choice for the interval will be $I=\left[0, T_{\infty}\right)$ where $T_{\infty} \in \mathbb{R} \cup\{+\infty\}$. A mapping $\gamma: I \rightarrow \mathbb{R}^{n}$ is referred in the sequel as a curve. Although the usual definition of a curve comes along with continuity and injectivity requirements for the map $\gamma$, we do not make these prior assumptions here. By the term continuous (respectively, absolutely continuous, Lipschitz, smooth) curve we shall refer to the corresponding properties of the mapping $\gamma: I \rightarrow \mathbb{R}^{n}$. A curve $\gamma$ is said to be bounded if its image, denoted by $\Gamma=\gamma(I)$, is a bounded set of $\mathbb{R}^{n}$.

The length of a curve $\gamma: I \rightarrow \mathbb{R}^{n}$ is defined as

$$
\ell(\gamma):=\sup \left\{\sum_{i=0}^{m-1} d\left(\gamma\left(t_{i}\right), \gamma\left(t_{i+1}\right)\right)\right\},
$$

where the supremum is taken over all finite increasing sequences $t_{0}<t_{1}<\cdots<t_{m}$ that lie in the interval $I$. Notice that $\ell(\gamma)$ corresponds to the total variation of the function $\gamma: I \rightarrow \mathbb{R}^{n}$. Let us mention for completeness that the length $\ell(\gamma)$ of a continuous injective curve $\gamma$ is equal to the unidimensional Hausdorff measure $\mathcal{H}^{1}(\Gamma)$ of its image, see e.g. [3, Th.2.6.2], but it is in general greater for noncontinuous curves. In particular we emphasis that for a piecewise continuous curve, the quantity $\ell(\gamma)$ is strictly greater than the sum of the lengths of each pieces but we still call $\ell(\gamma)$ the length of $\gamma$. A curve is called rectifiable, if it has locally bounded length.

Let us finally define the width of a (nonempty) convex subset $K$ of $\mathbb{R}^{n}$ at the direction $u \in \mathbb{S}^{n-1}$ as being the length of its orthogonal projection $P_{u}(K)$ on the 1-dimensional space $\mathbb{R} u$ generated by $u$. The following definition will play a key role in our main result.

Definition 2.1 (Mean width). The mean width of a nonempty convex set $K \subset \mathbb{R}^{n}$ is given by the formula

$$
W(K)=\frac{1}{\sigma_{n}} \int_{\mathbb{S}^{n-1}} \mathscr{L}^{1}\left(P_{u}(K)\right) d u,
$$

where $d u$ denotes the standard volume form on $\mathbb{S}^{n-1}$, and

$$
\sigma_{n}=\int_{\mathbb{S}^{n-1}} d u=\frac{n \pi^{n / 2}}{\Gamma\left(\frac{n}{2}+1\right)} .
$$

2.1. Self-expanded curves. Let us now recall from [13] the definition and the basic properties of a favorable class of Lipschitz curves, which has been studied thereby without a specific name. In the sequel we call these curves self-expanded.

Definition 2.2 (Self-expanded curve). A Lipschitz curve $\gamma: I \rightarrow \mathbb{R}^{n}$ is called self-expanded curve if for every $t \in I$ such that $\gamma^{\prime}(t)$ exists, we have that $\left\langle\gamma^{\prime}(t), \gamma(t)-\gamma(u)\right\rangle \geq 0$ for all $u \in I$ such that $u \leq t$.

In [13, 3.IX.] the following result has been established concerning self-expanded curves.

Theorem 2.3 ([13, 3.IX.]). Let $\gamma: I \rightarrow \mathbb{R}^{n}$ be a self-expanded curve of finite length. Then there exists a constant $C>0$ depending only on the dimension $n$ such that

$$
\ell(\gamma) \leq C \operatorname{diam} K
$$

where $K$ is any compact set containing $\Gamma=\gamma(I)$.

Notice that, formally, the above result requires the curve to have finite length. Nevertheless, the following limiting argument allows to obtain a more general conclusion for bounded selfexpanded curves. 
Corollary 2.4 (Bounded self-expanded curves have finite length). Every bounded self-expanded curve $\gamma: I \rightarrow \mathbb{R}^{n}$ has finite length and 2.3 holds.

Proof. Since self-expanded curves are rectifiable by definition, and because reparameterizing $\gamma$ does not change the statement, we may assume that $\gamma$ is parameterized by its arc-length on $I=[0, \ell(\gamma))$. Notice though that in principle $\ell(\gamma)$ might be infinite. Our aim is precisely to show that this is not the case. Indeed, let $K$ be a compact set containing $\gamma(I)$ and let $\left\{L_{n}\right\}_{n}$ be an increasing sequence of real numbers converging to $\ell(\gamma) \in \mathbb{R} \cup\{+\infty\}$. Applying Theorem 2.3 for the curve $\gamma_{n}:\left[0, L_{n}\right] \rightarrow \mathbb{R}^{n}$ (restriction of $\gamma$ to $\left[0, L_{n}\right]$ ), we obtain

$$
L_{n}=\ell\left(\gamma_{n}\right) \leq C \operatorname{diam} K, \quad \text { for all } n \geq 1 .
$$

This shows that $\ell(\gamma)=\lim _{n \rightarrow+\infty} L_{n}$ is bounded and satisfies the same estimate.

2.2. Self-contracted versus self-expanded curves. The aim of this section is to prove that Lipschitz continuous self-contracted curves and self-expanded curves give rise to the same images. Moreover, each of these curves can be obtained from the other upon reparameterization (inverting orientation). As a byproduct, bounded Lipschitz self-contracted curves in $\mathbb{R}^{n}$ have finite length.

Let us recall the definition of a self-contracted curve (see [6, Definition 1.2]).

Definition 2.5 (Self-contracted curve). A curve $\gamma: I \rightarrow \mathbb{R}^{n}$ is called self-contracted, if for every $t_{1} \leq t_{2} \leq t_{3}$ in $I$ we have

$$
d\left(\gamma\left(t_{1}\right), \gamma\left(t_{3}\right)\right) \geq d\left(\gamma\left(t_{2}\right), \gamma\left(t_{3}\right)\right) .
$$

In other words, the function $t \mapsto d\left(\gamma(t), \gamma\left(t_{3}\right)\right)$ is nonincreasing on $I \cap\left(-\infty, t_{3}\right]$.

Remark 2.6. (i) As we already said before, the definition of self-contracted curve can be given in any metric space and does not require any regularity of the curve, such as continuity or differentiability. Notice moreover that if $\gamma\left(t_{1}\right)=\gamma\left(t_{3}\right)$ in 2.4 above, then $\gamma(t)=\gamma\left(t_{1}\right)$ for $t_{1} \leq t \leq t_{2}$; thus if $\gamma$ is not locally stationary, then it is injective.

(ii) It has been proved in [6, Proposition 2.2] that if $\gamma$ is self-contracted and bounded, and $I=\left[0, T_{\infty}\right)$ with $T_{\infty} \in \mathbb{R}^{+} \cup\{+\infty\}$, then $\gamma$ converges to some point $\gamma_{\infty} \in \mathbb{R}^{n}$ as $t \rightarrow T_{\infty}$. (Notice that this conclusion follows also from our main result Theorem 3.3.) Consequently the curve $\gamma$ can be extended to $\bar{I}=\left[0, T_{\infty}\right]$. In particular, if $\gamma$ is continuous, then denoting by $\Gamma=\gamma(I)$ the image of $\gamma$, it follows that the set

$$
\bar{\Gamma}=\gamma(I) \cup\left\{\gamma_{\infty}\right\}=\gamma(\bar{I})
$$

is a compact subset of $\mathbb{R}^{n}$.

Lemma 2.7 (Property of a differentiability point). Let $\gamma: I \rightarrow \mathbb{R}^{n}$ be a self-contracted curve and let $t$ be a point of differentiability of $\gamma$. Then

$$
\left\langle\gamma^{\prime}(t), \gamma(u)-\gamma(t)\right\rangle \geq 0 \text { for all } u \in I \text { such that } u>t .
$$

Proof. Assume that $\gamma$ is differentiable at $t \in I$, and write $\gamma(t+s)=\gamma(t)+s \gamma^{\prime}(t)+o(s)$, with $\lim _{s \rightarrow 0} s^{-1} o(s)=0$. Let $u \in I$ be such that $u>t$, take $s$ such that $0<s<u-t$ and apply (2.4) for $t_{1}=t, t_{2}=t+s$ and $t_{3}=u$. We deduce that

$$
\|\gamma(t)-\gamma(u)\| \geq\|\gamma(t+s)-\gamma(u)\| .
$$


Since $\gamma(t+s)-\gamma(u)=\gamma(t)-\gamma(u)+s \gamma^{\prime}(t)+o(s)$, substituting this in the above inequality and squaring yields

$$
\begin{aligned}
0 & \geq\|\gamma(t+s)-\gamma(u)\|^{2}-\|\gamma(t)-\gamma(u)\|^{2} \\
& =2\left\langle s \gamma^{\prime}(t)+o(s), \gamma(t)-\gamma(u)\right\rangle+\left\|s \gamma^{\prime}(s)+o(s)\right\|^{2} \\
& =2 s\left\langle\gamma^{\prime}(t), \gamma(t)-\gamma(u)\right\rangle+o(s) .
\end{aligned}
$$

Dividing by $s$, and taking the limit as $s$ tends to $0^{+}$we get the desired result.

Given a curve $\gamma: I \mapsto \mathbb{R}^{n}$, we denote by $I^{-}=-I=\{-t ; t \in I\}$ the opposite interval, and define the reverse parametrization $\gamma^{-}: I^{-} \mapsto \mathbb{R}^{n}$ of $\gamma$ by $\gamma^{-}(t)=\gamma(-t)$ for $t \in I^{-}$.

Lemma 2.8 (Lipschitz self-contracted versus self-expanded curves). Let $\gamma: I \rightarrow \mathbb{R}^{n}$ be a Lipschitz curve. Then $\gamma$ is self-contracted if and only if $\gamma^{-}$is a self-expanded curve.

Proof. If $\gamma: I \rightarrow \mathbb{R}^{n}$ is Lipschitz and self-contracted, then Lemma 2.7 applies, yielding directly that $\gamma^{-}$is a self-expanded curve. Conversely, suppose that $\gamma^{-}$is a self-expanded curve. This means that $\gamma$ is Lipschitz and (after reversing the orientation) that

$$
\left\langle\gamma^{\prime}(t), \gamma(u)-\gamma(t)\right\rangle \geq 0 \text { for } u \in I \text { such that } u>t
$$

whenever $\gamma^{\prime}(t)$ exists and is different from 0 .

Fix now any $t_{3} \in I$ and define the function

$$
f(t)=\frac{1}{2}\left\|\gamma(t)-\gamma\left(t_{3}\right)\right\|^{2}, \quad \text { for all } t \in I, t \leq t_{3} .
$$

By Rademacher's theorem the Lipschitz continuous functions $\gamma$ is differentiable $\mathscr{L}^{1}$-almost everywhere, and so $f$ is too, and $f^{\prime}(t)=\left\langle\gamma^{\prime}(t), \gamma(t)-\gamma\left(t_{3}\right)\right\rangle$ for almost all $t \in I \cap\left(-\infty, t_{3}\right)$.

If $t<t_{3}$ and $\gamma^{\prime}(t) \neq 0$ then (2.5) above (for $u=t_{3}$ ) yields that $f^{\prime}(t) \leq 0$. Otherwise, $f^{\prime}(t)=0$. It follows that the Lipschitz function $f$ satisfies $f^{\prime}(t) \leq 0 \mathscr{L}^{1}$-almost everywhere, thus it is nondecreasing. This establishes (2.4). Since $t_{3}$ has been chosen arbitrarily, the proof is complete.

\section{RECTIFIABILITY OF SELF-CONTRACTED CURVES}

In [6, Theorem 1.3] it has been established that bounded self-contracted continuous planar curves $\gamma:[0,+\infty) \rightarrow \mathbb{R}^{2}$ have finite length. In this section we improve this result by dropping the continuity assumption, and we extend it to any dimension. Precisely, we establish that the length of any self-contracted curve $\gamma: I \rightarrow \mathbb{R}^{n}$ lying inside a compact set is bounded by a quantity depending only on the dimension of the space and the diameter of the compact set, see the forthcoming Theorem 3.3 .

3.1. Proof of the main result. The proof makes use of the following technical facts.

Lemma 3.1 (Saturating the sphere). Let $\Sigma \subseteq \mathbb{S}^{n-1}$ be such that $\langle x, y\rangle \leq 1 / 2$ for all $x, y \in$ $\Sigma, x \neq y$. Then $\Sigma$ is finite and $\sharp \Sigma \leq 3^{n}$.

Proof. For any $x, y \in \Sigma, x \neq y$, we have $\|x-y\|^{2}=2-2\langle x, y\rangle \geq 1$. Therefore, the open balls $\{B(x, 1 / 2)\}_{x \in \Sigma}$ are disjoint and they are all contained in the ball $B\left(0, \frac{3}{2}\right)$. Set $\omega_{n}=\mathscr{L}^{n}(B(0,1))$ (the measure of the unit ball); then

$$
(\sharp \Sigma) \omega_{n}\left(\frac{1}{2}\right)^{n}=\mathscr{L}^{n}\left(\bigcup_{x \in \Sigma} B(x, 1 / 2)\right) \leq \mathscr{L}^{n}(B(0,3 / 2))=\omega_{n}\left(\frac{3}{2}\right)^{n}
$$


and so $\sharp \Sigma \leq 3^{n}$.

Lemma 3.2 (Hemisphere lemma). Let $\Sigma \subset \mathbb{S}^{n-1}$ be a set satisfying

$$
\langle x, y\rangle \geq-\left(\frac{1}{3}\right)^{n+1} \text { for all } x, y \in \Sigma .
$$

Then there exists $\zeta \in \mathbb{S}^{n-1}$ such that

$$
\langle\zeta, x\rangle \geq\left(\frac{1}{3}\right)^{2 n+1} \quad \text { for all } x \in \Sigma .
$$

Proof. Let $\left\{x_{i}\right\}_{i \in I}$ be a family of points in $\Sigma$, maximal with respect to the property that

$$
\left\langle x_{i}, x_{j}\right\rangle \leq \frac{1}{2} \text { for all } i \neq j \text { in } I \text {. }
$$

(Notice that such a family can easily be constructed by induction.) Applying Lemma 3.1 we deduce that $m:=\sharp I \leq 3^{n}$. Set

$$
v:=\sum_{i \in I} x_{i}
$$

and let $y \in \Sigma$ be an arbitrary point. If $y$ does not belong to the family $\left\{x_{i}\right\}_{i \in I}$ then by maximality of the latter, there exists some $i_{0}$ such that $\left\langle x_{i_{0}}, y\right\rangle>\frac{1}{2}$. If on the contrary, $y=x_{i}$ for some $i$, then we take $i_{0}=i$. In view of (3.1) we obtain

$$
\langle v, y\rangle=\left\langle x_{i_{0}}, y\right\rangle+\sum_{i \neq i_{0}}\left\langle x_{i}, y\right\rangle \geq \frac{1}{2}-(m-1)\left(\frac{1}{3}\right)^{n+1},
$$

whence, recalling that $m \leq 3^{n}$, we get $\langle y, v\rangle \geq 3^{-(n+1)}$. This shows in particular that $v \neq 0$. Let us now compute $\|v\|$. We write

$$
\|v\|^{2}=\left\|\sum_{i \in I} x_{i}\right\|^{2}=\sum_{i \in I}\left\|x_{i}\right\|^{2}+E=m+E
$$

where

$$
E=\sum_{i \in I} \sum_{j \neq i}\left\langle x_{i}, x_{j}\right\rangle .
$$

Using (3.2) we deduce that $|E| \leq m(m-1) / 2$. Therefore (3.4) yields

$$
\|v\|^{2} \leq m+\frac{m(m-1)}{2}=\frac{m}{2}(m+1) \leq \frac{3^{n}}{2}\left(3^{n}+1\right) \leq 3^{2 n} .
$$

So dividing by $\|v\|$ in 3.3 and setting

$$
\zeta:=\frac{v}{\|v\|} \in \mathbb{S}^{n-1}
$$

we obtain

$$
\langle\zeta, y\rangle=\frac{1}{\|v\|}\langle v, y\rangle \geq\left(\frac{1}{3}\right)^{n}\left(\frac{1}{3}\right)^{n+1}=\left(\frac{1}{3}\right)^{2 n+1} .
$$

Since $y$ is arbitrary in $\Sigma$, the proof is complete.

We are now ready to prove the main result of this section. 
Theorem 3.3. Let $\gamma: I \rightarrow \mathbb{R}^{n}$ be a self-contracted curve. Then there exists a constant $C_{n}$ (depending only on the dimension $n$ ) such that

$$
\ell(\gamma) \leq C_{n} W(K)
$$

where $K$ is the closed convex hull of $\gamma(I)$. In particular, bounded self-contracted curves have finite length.

Proof. The result holds vacuously for unbounded curves (both left-hand and right-hand side of (3.5) are equal to $+\infty$ ). Therefore, we focus our attention on bounded self-contracted curves and assume that $K$ is compact. We may also clearly assume that $n \geq 2$ (the result is trivial in the one-dimensional case).

In the sequel, we denote by $\Gamma=\gamma(I)$ the image of such curve. The set $\Gamma$ inherits from $I$ a total order as follows: for $x, y \in \Gamma$ we say that " $x$ is before $y$ " and denote $x \preceq y$, if there exist $t_{1}, t_{2} \in I, t_{1} \leq t_{2}$ and $\gamma\left(t_{1}\right)=x, \gamma\left(t_{2}\right)=y$. If $x \preceq y$ and $x \neq y$, then the intervals $\gamma^{-1}(x)$ and $\gamma^{-1}(y)$ do not meet, and for any $t_{1} \in \gamma^{-1}(x), t_{2} \in \gamma^{-1}(y)$ we have $t_{1}<t_{2}$. In this case we say that " $x$ is strictly before $y$ " and we denote $x \prec y$. For $x \in \Gamma$ we set

$$
\Gamma(x):=\{y \in \Gamma: x \preceq y\}
$$

(the piece of curve after $x$ ) and denote by $\Omega(x)$ the closed convex hull of $\Gamma(x)$.

Claim 1. To establish (3.5) it suffices to find a positive constant $\varepsilon=\varepsilon(n)$, depending only on the dimension $n$, such that for any two points $x, x^{\prime} \in \Gamma$ with $x^{\prime} \preceq x$ it holds

$$
W(\Omega(x))+\varepsilon\left\|x-x^{\prime}\right\| \leq W\left(\Omega\left(x^{\prime}\right)\right) .
$$

Proof of Claim 1. Let us see how we can deduce Theorem 3.3 from the above. To this end, let $t_{0}<t_{1} \ldots<t_{m}$ be any increasing sequence in $I$, and set $x_{i}=\gamma\left(t_{i}\right)$. If (3.6) holds, then

$$
\begin{aligned}
\sum_{i=0}^{m-1}\left\|\gamma\left(t_{i+1}\right)-\gamma\left(t_{i}\right)\right\| & =\sum_{i=0}^{m-1}\left\|x_{i+1}-x_{i}\right\| \leq \frac{1}{\varepsilon} \sum_{i=0}^{m-1}\left(W\left(C\left(x_{i}\right)-W\left(C\left(x_{i+1}\right)\right)\right)\right. \\
& =\frac{1}{\varepsilon}\left(W\left(C\left(x_{0}\right)\right)-W\left(C\left(x_{m}\right)\right)\right) \leq \frac{1}{\varepsilon} W\left(C\left(x_{0}\right)\right) \leq \frac{1}{\varepsilon} W(K),
\end{aligned}
$$

since the mean width $W(H)$ is a nondecreasing function of $H$ (the variable $H$ is ordered via the set inclusion). Taking the supremum over all choices of $t_{0}<t_{1} \ldots<t_{m}$ in $I$ we obtain (3.5) for $C_{n}=1 / \varepsilon$.

Therefore, the theorem will be proved, if we show that $(3.6)$ holds for some constant $\varepsilon>0$ which depends only on the dimension. Before we proceed, we introduce some extra notation. Given $x, x^{\prime}$ in $\Gamma$ with $x^{\prime} \prec x$ we set

$$
x_{0}:=\frac{1}{3} x+\frac{2}{3} x^{\prime} \text { and } v_{0}:=\frac{x^{\prime}-x}{\left\|x^{\prime}-x\right\|} .
$$

For the sake of drawing pictures, the reader is invited to think that $v_{0}=e_{1}$ (the first vector of the canonical basis of $\mathbb{R}^{n}$ ), see Figure 1 . Let us also set

$$
\xi_{0}(y)=\frac{y-x_{0}}{\left\|y-x_{0}\right\|} \in \mathbb{S}^{n-1}, \quad \text { for any } y \in \Gamma(x) .
$$

Clearly, $x_{0}, v_{0}$ and $\xi_{0}(y)$ depend on the points $x, x^{\prime}$, while the desired constant $\varepsilon$ does not. To determine this constant, we shall again transform the problem into another one (see the forthcoming Claim 2). 


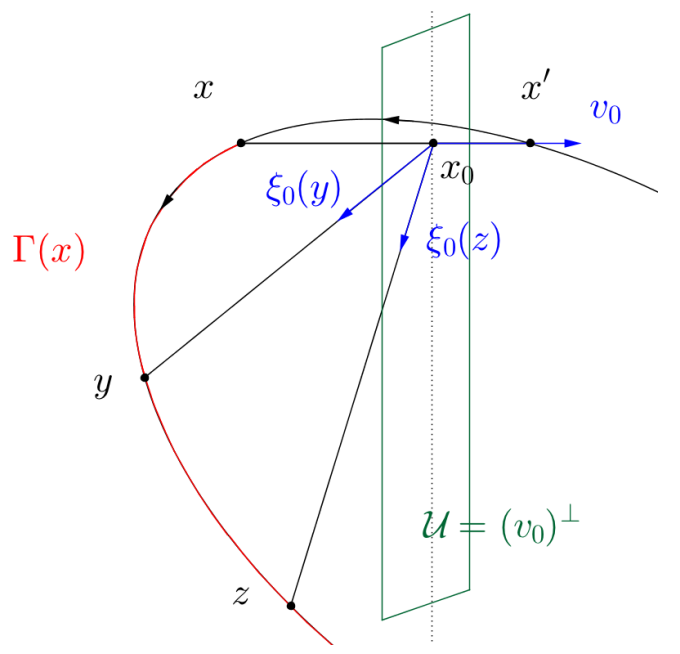

Figure 1. Controlling the tail of a self-contracted curve.

Claim 2. Let us assume that there exists a constant $0<\delta<2^{-4}$, depending only on the dimension $n$, such that for all $x, x^{\prime}$ in $\Gamma$ with $x^{\prime} \prec x$ (and for $x_{0}, v_{0}$ defined by (3.7)), there exists $\bar{v} \in \mathbb{S}^{n-1} \cap B\left(v_{0}, \delta\right)$ such that

$$
\left\langle\bar{v}, \xi_{0}(y)\right\rangle \leq-\delta^{2} \quad \text { for all } y \in \Gamma(x) .
$$

Then (3.6) holds true (and consequently (3.5) follows).

Proof of Claim 2. Assume that such a constant $\delta$ and a vector $\bar{v}$ exist, so that $(3.9)$ holds. Set

$$
V=\left\{v \in \mathbb{S}^{n-1} ;\|v-\bar{v}\| \leq \delta^{2}\right\} .
$$

Combining with 3.8 and $(3.9)$ we get

$$
\left\langle v, y-x_{0}\right\rangle \leq 0, \quad \text { for all } v \in V \text { and } y \in \Gamma(x) .
$$

Let us first explain intuitively why the above yields (3.6). Indeed, compared with $\Omega(x), \Omega\left(x^{\prime}\right)$ has an extra piece coming from the segment $\left[x_{0}, x^{\prime}\right]$. This piece is protruding in all directions $v \in V$ (which are relatively close to $v_{0}$ ), while (3.10) bounds uniformly the orthogonal projections of $\Omega(x)$ onto the lines $\mathbb{R} v$. Therefore, the extra contribution of the segment $\left[x_{0}, x^{\prime}\right]$ in $P_{v}\left(\Omega\left(x^{\prime}\right)\right)$ becomes perceptible and can be quantified in terms of $\left\|x_{0}-x^{\prime}\right\|$, uniformly in $V$. Since the latter set $V$ has a positive measure, the estimation (3.6) follows.

To proceed, observe that $\Omega\left(x^{\prime}\right)$ contains the convex hull of $\Omega(x) \cup\left[x, x^{\prime}\right]$, whence

$$
P_{v}(\Omega(x)) \subset P_{v}\left(\Omega\left(x^{\prime}\right)\right) .
$$

In particular,

$$
\mathcal{H}^{1}\left(P_{v}(\Omega(x))\right) \leq \mathcal{H}^{1}\left(P_{v}\left(\Omega\left(x^{\prime}\right)\right)\right) \text { for } v \in \mathbb{S}^{n-1},
$$

where $P_{v}$ denotes the orthogonal projection onto the line $\mathbb{R} v$. Let us now equip the latter with the obvious order (stemming from the identification $\mathbb{R} v \cong \mathbb{R}$ ) and let us identify $P_{v}\left(x_{0}\right)$ with the zero element 0 . Then (3.10) says that for all directions $v$ in $V$ we have

$$
\sup P_{v}(\Omega(x)) \leq 0<P_{v}\left(x^{\prime}\right) \leq \sup P_{v}\left(\Omega\left(x^{\prime}\right)\right) .
$$

Notice that

$$
\left\|x_{0}-x^{\prime}\right\|=\frac{1}{3}\left\|x-x^{\prime}\right\|
$$


and that $V \subset B\left(v_{0}, \delta+\delta^{2}\right)$. Thus for every $v \in V$ we have

$$
\left\langle v_{0}, v\right\rangle \geq\left\langle v_{0}, v_{0}\right\rangle-\left\|v_{0}\right\|\left\|v_{0}-v\right\| \geq 1-\delta-\delta^{2} \geq 7 / 8
$$

This gives a lower bound for the length of the projected segment $\left[x_{0}, x^{\prime}\right]$ onto $\mathbb{R} v$, which coincides, under the above identification, with $P_{v}\left(x^{\prime}\right)$. Thus

$$
P_{v}\left(x^{\prime}\right) \geq \frac{7}{8}\left(\frac{1}{3}\left\|x-x^{\prime}\right\|\right)>\frac{1}{4}\left\|x-x^{\prime}\right\| .
$$

This yields

$$
\mathcal{H}^{1}\left(P_{v}(\Omega(x))\right)+\frac{1}{4}\left\|x-x^{\prime}\right\| \leq \mathcal{H}^{1}\left(P_{v}\left(\Omega\left(x^{\prime}\right)\right)\right) \text { for } v \in V .
$$

Integrating (3.12) for $v \in V$ and (3.11) for $v \in \mathbb{S}^{n-1} \backslash V$, and summing up the resulting inequalities we obtain $(3.6)$ with

$$
\varepsilon=\left(4 \sigma_{n}\right)^{-1} \int_{V} d u
$$

Notice that this bound only depends on $\delta$, so the claim follows.

Consequently, our next goal is to determine $\delta>0$ so that the assertion of Claim 2 holds. The exact value of the parameter $\delta$ is eventually given in (3.25) and depends only on the dimension. In particular, it works for any self-contracted curve and any choice of points $x, x^{\prime} \in \Gamma$. For the remaining part of the proof, it is possible to replace $\delta$ by its precise value. Nevertheless, we prefer not to do so, in order to illustrate how this value is obtained. In the sequel, the only prior requirement is the bound $\delta \leq 2^{-4}$.

Fix any $x, x^{\prime}$ in $\Gamma$ with $x^{\prime} \prec x$ and recall the definition of $x_{0}, v_{0}$ in (3.7) and $\xi_{0}(y)$, for $y \in \Gamma(x)$ in (3.8). Based on this, we consider the orthogonal decomposition

$$
\mathbb{R}^{n} \approx \mathbb{R} v_{0} \oplus \mathcal{U}
$$

where $\mathcal{U}=\left(v_{0}\right)^{\perp}$ is the orthogonal hyperplane to $v_{0}$. The vector $\bar{v}$ of the assertion of Claim 2 will be taken of the form

$$
\bar{v}=\frac{v_{0}-\delta \zeta}{\left\|v_{0}-\delta \zeta\right\|}
$$

where $\zeta$ is a unit vector in $\mathcal{U}$. This vector will be determined later on, as an application of Lemma 3.2 notice however that for any $\zeta \in \mathbb{S}^{n-1} \cap \mathcal{U}$ we get $\bar{v} \in \mathbb{S}^{n-1} \cap B\left(v_{0}, \delta\right)$, as needed.

We set

$$
\Gamma_{0}=\left\{y \in \Gamma(x) ;\left\langle v_{0}, \xi_{0}(y)\right\rangle \leq-2 \delta\right\} .
$$

Notice that for every $y \in \Gamma_{0}$ and any $\bar{v}$ of the form (3.14) we have

$$
\left\langle\bar{v}, \xi_{0}(y)\right\rangle \leq-2 \delta+\left\|v_{0}-\bar{v}\right\| \leq-\delta \leq-\delta^{2} .
$$

Thus (3.9) is satisfied for all $y$ in $\Gamma_{0}$.

It remains to choose $\zeta$ (and adjust the value of $\delta$ ) so that (3.9) would also hold for $y \in \Gamma(x) \backslash \Gamma_{0}$. This will be done in five steps. In the sequel we shall make use of the decomposition (3.13) of vectors $\xi_{0}(y), y \in \Gamma(x)$, namely:

$$
\xi_{0}(y)=\left\langle v_{0}, \xi_{0}(y)\right\rangle v_{0}+\xi_{0}^{\mathcal{U}}(y),
$$

where $\xi_{0}^{\mathcal{U}}(y)$ is the orthogonal projection of $\xi_{0}(y)$ in $\mathcal{U}$. 
Step 1. We establish that for all $y \in \Gamma(x) \backslash\{x\}$ it holds

$$
\left\langle v_{0}, \xi_{0}(y)\right\rangle \leq-\frac{\left\|x-x^{\prime}\right\|}{6\left\|y-x_{0}\right\|}<0 .
$$

Indeed, since $x^{\prime} \prec x \prec y$, we deduce from self-contractedness that $\left\|y-x^{\prime}\right\| \geq\|y-x\|$, that is, $y$ lies at the same half-space as $x$ defined by the mediatrix hyperplane of the segment $\left[x, x^{\prime}\right]$. Hence, denoting by $P_{v_{0}}$ the orthogonal projection of $\mathbb{R}^{n}$ on $\mathbb{R} v_{0}$ (which we brutally identify to $\mathbb{R}$ to write inequalities) we observe that

$$
P_{v_{0}}(y) \leq \frac{1}{2} P_{v_{0}}(x)+\frac{1}{2} P_{v_{0}}\left(x^{\prime}\right)=P_{v_{0}}(x)+\frac{1}{2}\left\|x-x^{\prime}\right\|,
$$

and consequently

$$
\left\langle v_{0}, y-x_{0}\right\rangle=P_{v_{0}}\left(y-x_{0}\right) \leq P_{v_{0}}(x)+\frac{1}{2}\left\|x-x^{\prime}\right\|-P_{v_{0}}\left(x_{0}\right)=-\frac{1}{6}\left\|x-x^{\prime}\right\|,
$$

thus, dividing by $\left\|y-x_{0}\right\|$, (3.17) follows.

Step 2. We establish that for all $y \in \Gamma(x) \backslash \Gamma_{0}$, we have:

and

$$
\begin{gathered}
\left\|y-x_{0}\right\|>\frac{1}{12 \delta}\left\|x-x^{\prime}\right\| ; \\
\left|\left\langle v_{0}, \xi_{0}(y)\right\rangle\right|=\left\|\xi_{0}(y)-\xi_{0}^{\mathcal{U}}(y)\right\| \leq 2 \delta
\end{gathered}
$$

$$
\sqrt{1-4 \delta^{2}} \leq\left\|\xi_{0}^{\mathcal{U}}(y)\right\| \leq 1
$$

Indeed, since $y \in \Gamma(x) \backslash \Gamma_{0},(3.18)$ follows easily by combining (3.15) with (3.17). The same formulas yield that $\left\langle v_{0}, \xi_{0}(y)\right\rangle \in(-2 \delta, 0)$, whence $\left|\left\langle v_{0}, \xi_{0}(y)\right\rangle\right| \leq 2 \delta$. In view of (3.16), both (3.19) and (3.20) follow directly. This ends the proof of Step 2.

Before we proceed, let us make the following observation, which motivates Step 4 (compare forthcoming inequalities (3.22) and (3.24) ). We set

$$
\xi(y)=\frac{y-x}{\|y-x\|} \in \mathbb{S}^{n-1}, \quad \text { for all } y \in \Gamma(x) \backslash\{x\} .
$$

It is easily seen that

$$
\langle\xi(y), \xi(z)\rangle \geq 0, \quad \text { for all } y, z \in \Gamma(x) \backslash\{x\} .
$$

Indeed assuming $x \preceq y \prec z$ (the case $y=z$ is trivial), the definition of self-contractedness yields

$$
\|z-x\| \geq\|z-y\| \text {. }
$$

Writing $z-y=(z-x)-(y-x)$ and squaring the above inequality, we obtain

$$
\|z-x\|^{2} \geq\|z-x\|^{2}+\|x-y\|^{2}-2\langle z-x, y-x\rangle,
$$

which yields $\langle z-x, y-x\rangle \geq 0$. Dividing by norms gives 3.22 .

Our next objective is to show that vectors $\xi_{0}^{\mathcal{U}}(y), y \in \Gamma(x) \backslash \Gamma_{0}$ satisfy a relaxed inequality of type 3.22 . We need the following intermediate step.

Step 3. We show that for every $y \in \Gamma(x) \backslash \Gamma_{0}$, we have:

$$
\left\|\xi(y)-\xi_{0}(y)\right\| \leq 32 \delta .
$$


Indeed

$$
\begin{aligned}
\left\|\xi(y)-\xi_{0}(y)\right\| & \leq\left\|\frac{y-x}{\|y-x\|}-\frac{y-x_{0}}{\|y-x\|}\right\|+\left\|\frac{y-x_{0}}{\|y-x\|}-\frac{y-x_{0}}{\left\|y-x_{0}\right\|}\right\| \\
& =\frac{\left\|x-x_{0}\right\|}{\|y-x\|}+\left|\frac{1}{\|y-x\|}-\frac{1}{\left\|y-x_{0}\right\|}\right|\left\|y-x_{0}\right\| \\
& \leq \frac{2\left\|x-x_{0}\right\|}{\|y-x\|}=\frac{4}{3} \frac{\left\|x-x^{\prime}\right\|}{\|y-x\|} .
\end{aligned}
$$

On the other hand, using (3.18) we get:

$$
\|y-x\| \geq\left\|y-x_{0}\right\|-\left\|x_{0}-x\right\| \geq\left(\frac{1}{12 \delta}-\frac{2}{3}\right)\left\|x-x^{\prime}\right\|=\left(\frac{1-8 \delta}{16 \delta}\right)\left(\frac{4\left\|x-x^{\prime}\right\|}{3}\right) .
$$

Combining the above inequalities, we deduce

$$
\left\|\xi(y)-\xi_{0}(y)\right\| \leq \frac{16 \delta}{1-8 \delta},
$$

thus 3.23 follows thanks to the prior bound $\delta \leq 2^{-4}$.

Step 4. We now establish that for every $y, z \in \Gamma(x) \backslash \Gamma_{0}$, we have:

$$
\left\langle\xi_{0}^{\mathcal{U}}(y), \xi_{0}^{\mathcal{U}}(z)\right\rangle \geq-65 \delta .
$$

Let us first calculate the scalar product of the unit vectors $\xi_{0}(y)$ and $\xi_{0}(z)$.

$$
\begin{aligned}
\left\langle\xi_{0}(y), \xi_{0}(z)\right\rangle & =\left\langle\xi(y), \xi_{0}(z)\right\rangle+\left\langle\xi_{0}(y)-\xi(y), \xi_{0}(z)\right\rangle \\
& =\langle\xi(y), \xi(z)\rangle+\left\langle\xi(y), \xi_{0}(z)-\xi(z)\right\rangle+\left\langle\xi_{0}(y)-\xi(y), \xi_{0}(z)\right\rangle \\
& \geq 0-\left\|\xi_{0}(z)-\xi(z)\right\|-\left\|\xi_{0}(y)-\xi(y)\right\| \geq-64 \delta,
\end{aligned}
$$

where $(3.22)$ and $(3.23)$ have been used for the last estimation.

Using now the above estimation, the orthogonal decomposition $(3.16)$ recalling $(3.19)$ and the prior bound $\delta \leq 2^{-4}$ we obtain successively:

$$
\begin{aligned}
\left\langle\xi_{0}^{\mathcal{U}}(y), \xi_{0}^{\mathcal{U}}(z)\right\rangle & =\left\langle\xi_{0}(y), \xi_{0}(z)\right\rangle-\left\langle v_{0}, \xi_{0}(y)\right\rangle\left\langle v_{0}, \xi_{0}(z)\right\rangle \\
& \geq-64 \delta-4 \delta^{2} \geq-65 \delta .
\end{aligned}
$$

Step 5. We shall now be interested in the set

$$
\Sigma=\left\{\hat{\xi}_{0}^{\mathcal{U}}(y) ; y \in \Gamma(x) \backslash \Gamma_{0}\right\} \subset \mathcal{U} \cap \mathbb{S}^{n-1},
$$

where $\hat{\xi}_{0}^{\mathcal{U}}(y)=\xi_{0}^{\mathcal{U}}(y) /\left\|\xi_{0}^{\mathcal{U}}(y)\right\|$ (notice that $\xi_{0}^{\mathcal{U}}(y) \neq \emptyset$ from $(3.20)$ ). In view of (3.24) and the estimation (3.20), we obtain that for all $y, z \in \Gamma(x) \backslash \Gamma_{0}$

$$
\left\langle\hat{\xi}_{0}^{\mathcal{U}}(y), \hat{\xi}_{0}^{\mathcal{U}}(z)\right\rangle \geq \frac{-65 \delta}{1-4 \delta^{2}} \geq-81 \delta
$$

We are now ready to give a precise value for $\delta$, namely,

$$
\delta=\left(\frac{1}{3}\right)^{3 n}
$$

Recalling that $n \geq 2$, under this choice the scalar product between two elements of $\Sigma$ is bounded from below by the quantity

$$
-81 \delta \geq-81\left(\frac{1}{3}\right)^{3 n} \geq-\left(\frac{1}{3}\right)^{n} .
$$


Therefore, the subset $\Sigma$ of the unit sphere of $\mathcal{U} \simeq \mathbb{R}^{n-1}$ satisfies the assumptions of Lemma 3.2. We deduce that there exists a unit vector $\zeta \in \mathcal{U} \cap \mathbb{S}^{n-1}$ such that

$$
\left\langle\zeta, \hat{\xi}_{0}^{\mathcal{U}}(y)\right\rangle \geq\left(\frac{1}{3}\right)^{2 n-1}, \text { for all } y \in \Gamma(x) \backslash \Gamma_{0} .
$$

Taking $\delta$ and $\zeta$ as above in (3.14), we obtain $\bar{v} \in \mathbb{S}^{n-1} \cap B\left(v_{0}, \delta\right)$. Let us now verify that (3.9) holds. As already mentioned (see comments before Step 1), it remains to consider the case $y \in \Gamma(x) \backslash \Gamma_{0}$. Notice that in view of (3.17)

$$
\left\langle v_{0}-\delta \zeta, \xi_{0}(y)\right\rangle=\left\langle v_{0}, \xi_{0}(y)\right\rangle-\delta\left\langle\zeta, \xi_{0}(y)\right\rangle \leq-\delta\left\langle\zeta, \xi_{0}(y)\right\rangle,
$$

while in view of $(3.13),(3.20)$ and the orthogonality between $v_{0}$ and $\zeta$ we get

$$
\left\langle\zeta, \xi_{0}(y)\right\rangle=\left\langle\zeta, \xi_{0}^{\mathcal{U}}(y)\right\rangle=\left\|\xi_{0}^{\mathcal{U}}(y)\right\|\left\langle\zeta, \hat{\xi}_{0}^{\mathcal{U}}(y)\right\rangle \geq \sqrt{1-4 \delta^{2}}\left(\frac{1}{3}\right)^{2 n-1} \geq\left(\frac{1}{3}\right)^{2 n} .
$$

Assembling the above, and using the fact that $\left\|v_{0}-\delta \zeta\right\|=\sqrt{1+\delta^{2}}>1$ we obtain

$$
\left\langle\bar{v}, \xi_{0}(y)\right\rangle \leq-\delta \sqrt{1+\delta^{2}}\left(\frac{1}{3}\right)^{2 n} \leq-\delta\left(\frac{1}{3}\right)^{3 n}=-\delta^{2} .
$$

This shows that the assumption made in Claim 2 is always fulfilled. The proof is complete.

3.2. Arc-length reparameterization. Having in hand that the length of a bounded selfcontracted curve $\gamma: I \rightarrow \mathbb{R}^{n}$ is finite (c.f. Theorem 3.3), the curve $\gamma$ may be reparameterized by its arc-length. This reparameterization is particularly interesting when the curve $\gamma$ is continuous. In this case, we shall see that the arc-length parametrization is Lipschitz continuous and that the image $\Gamma=\gamma(I)$ of the curve can also be realized by a self-expanded curve.

Set $I(t)=I \cap(-\infty, t]$, for $t \in I$, and consider the length function

$$
\left\{\begin{array}{l}
L: I \rightarrow[0, \ell(\gamma)] \\
L(t)=\ell\left(\gamma_{\mid I(t)}\right)
\end{array}\right.
$$

Thus $L(t)$ is the length of the truncated curve $\left.\gamma\right|_{I(t)}$ and consequently the function $t \mapsto L(t)$ is nondecreasing, but not necessarily injective - it is locally constant whenever $t \mapsto \gamma(t)$ is locally stationary, see also Remark 2.6 (i). Let us assume that $\gamma$ is bounded and continuous. Then, in view of Remark 2.6 (ii), the curve can be extended continuously to $\bar{I}$ and the set $\bar{\Gamma}=\gamma(I) \cup\left\{\gamma_{\infty}\right\}=\gamma(\bar{I})$ is a compact connected arc. Moreover, $t \mapsto L(t)$ is continuous and $L(\bar{I})=[0, \ell(\gamma)]$. It follows easily that $L^{-1}(s)$ is a (possibly trivial) interval $\left[t_{1}, t_{2}\right]$. Consequently, the function

$$
\left\{\begin{array}{l}
\tilde{\gamma}:[0, \ell(\gamma)] \rightarrow \mathbb{R}^{n} \\
\tilde{\gamma}(s)=\gamma(t), \text { for any } t \in L^{-1}(s)
\end{array}\right.
$$

is well-defined.

Proposition 3.4 (Hidden regularity of continuous self-contracted curves). Let $\gamma: I \rightarrow \mathbb{R}^{n}$ be a bounded continuous self-contracted curve. Then (3.27) defines a Lipschitz self-contracted curve with the same image $\Gamma=\gamma(I)=\tilde{\gamma}([0, \ell(\gamma)))$. In particular, $\Gamma$ is also the image of some self-expanded curve.

Proof. Let $s, s^{\prime} \in[0, \ell(\gamma)]$ be given. Let $t, t^{\prime} \in I$ be such that $s=L(t)$ and $s^{\prime}=L\left(t^{\prime}\right)$. Assume that $s<s^{\prime}$ (thus a fortiori $t<t^{\prime}$ ). We claim that

$$
L\left(t^{\prime}\right) \geq L(t)+d\left(\gamma(t), \gamma\left(t^{\prime}\right)\right) .
$$


Indeed, recalling (2.2), for any $\varepsilon>0$, there exists a finite sequence $t_{0}<\ldots<t_{m}$ in $I(t)$, satisfying

$$
\Sigma_{1}=: \sum_{i=0}^{m-1} d\left(\gamma\left(t_{i}\right), \gamma\left(t_{i+1}\right)\right) \geq L(t)-\varepsilon .
$$

Since adding an extra point can only make the sum larger, we may assume that $t_{m}=t$. Then add the point $t_{m+1}=t^{\prime}$ and notice that $t_{0}<\ldots<t_{m}<t_{m+1}$ is a sequence in $I\left(t^{\prime}\right)$, thus

$$
\Sigma_{2}=: \sum_{i=0}^{m} d\left(\gamma\left(t_{i}\right), \gamma\left(t_{i+1}\right)\right)=\Sigma_{1}+d\left(\gamma(t), \gamma\left(t^{\prime}\right)\right) \leq L\left(t^{\prime}\right) \text {. }
$$

We deduce that

$$
L(t)-\varepsilon \leq \Sigma_{1}=\Sigma_{2}-d\left(\gamma(t), \gamma\left(t^{\prime}\right)\right) \leq L\left(t^{\prime}\right)-d\left(\gamma(t), \gamma\left(t^{\prime}\right)\right)
$$

Taking the limit as $\varepsilon \rightarrow 0$ and get (3.28). It follows that

$$
\left\|\tilde{\gamma}\left(s^{\prime}\right)-\tilde{\gamma}(s)\right\|=\left\|\gamma\left(t^{\prime}\right)-\gamma(t)\right\| \leq L\left(t^{\prime}\right)-L(t)=s^{\prime}-s,
$$

which proves that $\tilde{\gamma}$ is 1-Lipschitz on $[0, \ell(\gamma)]$.

It follows easily that $\tilde{\gamma}$ is self-contracted, while the fact that $\tilde{\gamma}([0, \ell(\gamma)))=\gamma(I)$ is straightforward. The last assertion follows from Lemma 2.8 .

Remark 3.5 (Improving the length bound). Although the constants $C>0$ in $(2.3)$ (for selfexpanded curves) and in (3.5) (for bounded self-contracted curves) stem from the same method, the latter is based on nonsmooth arguments encompassing nonregular curves and therefore it is not optimized. As a consequence of Proposition 3.4 given a continuous self-contracted curve we can use (2.3) to improve a posteriori the constant that bounds the length given in (3.5). Notwithstanding, this trick cannot be applied to any bounded self-contracted curve: in the discontinuous case, one can still consider the length mapping $L(t)$, and use it to construct a larger curve (whose image contains $\Gamma$ ) with a Lipschitz parametrization, but which is possibly not self-contracted.

\section{Applications}

4.1. Orbits of convex foliations. As mentioned in the introduction, self-contracted curves are naturally defined in a metric space without prior regularity assumptions. Nonetheless, this notion has been conceived to capture the behaviour of orbits of gradient dynamical systems for (smooth) quasiconvex potentials in Euclidean spaces (1.1), or more generally, orbits of the subgradient semi-flow defined by a nonsmooth convex function, see (1.2). In both cases, orbits are at least, absolutely continuous. In this section we broaden the above framework by introducing a new concept of generalized solution to a nonsmooth convex foliation, admitting (merely) continuous curves as generalized orbits.

4.1.1. Nonsmooth convex foliations. Let us first give the definition of a convex foliation.

Definition 4.1 (Convex foliation). A collection $\left\{C_{r}\right\}_{r \geq 0}$ of nonempty convex compact subsets of $\mathbb{R}^{n}$ is a (global) convex foliation of $\mathbb{R}^{n}$ if

$$
r_{1}<r_{2} \Longrightarrow C_{r_{1}} \subset \operatorname{int} C_{r_{2}}
$$

and

$$
\bigcup_{r \geq 0} \partial C_{r}=\mathbb{R}^{n} \backslash \operatorname{int} C_{0} .
$$


In view of (4.1), relation $(4.2)$ is equivalent to the fact that for every $x \in \mathbb{R}^{n} \backslash \operatorname{int} C_{0}$, there exists a unique $r \in[0, \infty)$ such that $x \in \partial C_{r}$. The above definition is thus equivalent to $[6$, Definition (6.5)].

Example 4.2 (Foliation given by a function). (i) The sublevel sets of a proper (coercive) convex function $f: \mathbb{R}^{n} \rightarrow \mathbb{R}$ provide a typical example of a convex foliation. Indeed, if $m=\min f$, we set $C_{0}=f^{-1}(m)$ and $C_{r}=[f \leq m+r]$. Then (4.2) follows from the fact that $f$ cannot be locally constant outside of $C_{0}$.

(ii) The sublevel sets of a (coercive) quasiconvex function might fail to satisfy 4.2 , , unless int $[f \leq \lambda]=[f<\lambda]$ for all $\lambda>\min f$. This condition is automatically satisfied if the quasiconvex function is smooth and every critical point is a global minimizer. A more general condition is to assume that $f$ is semistrictly quasiconvex, see for instance [5] for the relevant definition and characterizations.

4.1.2. Generalized solutions and self-contractedness. We consider a possibly unbounded interval $I=\left[0, T_{\infty}\right)$ of $\mathbb{R}$, where $T_{\infty} \in \mathbb{R}_{+} \cup\{+\infty\}$ and a continuous injective curve $\gamma: I \rightarrow \mathbb{R}^{n}$. For every $\tau \in I$ we define the set of all possible limits of backward secants at $\gamma(\tau)$ as follows:

$$
\sec ^{-}(\tau):=\left\{q \in \mathbb{S}^{n-1}: q=\lim _{t_{k} \nearrow^{-}} \frac{\gamma\left(t_{k}\right)-\gamma(\tau)}{\left\|\gamma\left(t_{k}\right)-\gamma(\tau)\right\|}\right\}
$$

where the notation $\left\{t_{k}\right\}_{k} \nearrow \tau^{-}$indicates that $\left\{t_{k}\right\}_{k} \rightarrow \tau$ and $t_{k}<\tau$ for all $k$. The set $\sec ^{+}(\tau)$ is defined analogously using decreasing sequences $\left\{t_{k}\right\}_{k} \searrow \tau^{+}$. The compactness of $\mathbb{S}^{n-1}$ guarantees that both $\sec ^{-}(\tau)$ and $\sec ^{+}(\tau)$ are nonempty. The following lemma relates $\sec ^{-}(\tau)$ and $\sec ^{+}(\tau)$ with the derivative of $\gamma$, in case the latter exists and does not vanish.

Lemma 4.3 (Secants versus derivative). Assume $\gamma: I \rightarrow \mathbb{R}^{n}$ is differentiable at $\tau \in I$ and $\gamma^{\prime}(\tau) \neq 0$. Then

$$
\sec ^{-}(\tau)=\left\{-\frac{\gamma^{\prime}(\tau)}{\left\|\gamma^{\prime}(\tau)\right\|}\right\} \quad \text { and } \quad \sec ^{+}(\tau)=\left\{\frac{\gamma^{\prime}(\tau)}{\left\|\gamma^{\prime}(\tau)\right\|}\right\}
$$

Proof. Let $q \in \sec ^{-}(\tau)$ and let $\left\{t_{k}\right\}_{k} \nearrow \tau^{-}$be a sequence realizing this limit. Then writing

$$
\frac{\gamma\left(t_{k}\right)-\gamma(\tau)}{\left\|\gamma\left(t_{k}\right)-\gamma(\tau)\right\|}=\left(\frac{\gamma\left(t_{k}\right)-\gamma(\tau)}{t_{k}-\tau}\right)\left(\frac{-\left|\tau-t_{k}\right|}{\left\|\gamma\left(t_{k}\right)-\gamma(\tau)\right\|}\right)
$$

and passing to the limit as $\left\{t_{k}\right\}_{k} \nearrow \tau^{-}$we obtain the result. The second assertion follows similarly.

Assuming $\gamma(t) \notin \operatorname{int} C_{0}$ we denote by $r(t)$ the unique positive number such that

$$
\gamma(t) \in \partial C_{r(t)}
$$

We are now ready to give the definition of generalized orbit to a convex foliation.

Definition 4.4 (Convex foliation orbits). A continuous curve $\gamma: I \rightarrow \mathbb{R}^{n}$ is called generalized solution (orbit) of the convex foliation $\left\{C_{r}\right\}_{r \geq 0}$ if $\gamma(I) \cap \operatorname{int} C_{0}=\emptyset$ and under the notation 4.3. and (4.4) we have:

(i) the function $t \mapsto r(t)$ is decreasing ;

(ii) $\sec ^{-}(t) \subset N_{C_{r(t)}}(\gamma(t))$ for all $t \in I$. 
It follows directly from (i) that $\gamma$ is injective, and then (ii) makes sense. Also, every orbit of a convex foliation is an injective mapping with bounded image $(\Gamma=\gamma(I)$ is contained in the compact set $\left.C_{r(0)}\right)$. Recall the definition of the normal cone $N_{C_{r(t)}}$ in $(2.1)$. If $\partial C_{r(t)}$ is a smooth manifold (or more generally, if $\operatorname{dim} N_{C_{r(t)}}(\gamma(t))=1$ ) we have $\sec ^{-}(t)=\left\{\nu_{r(t)}\right\}$ where $\nu_{r(t)}$ is the unit normal of $\partial C_{r(t)}$ at $\gamma(t)$.

In the sequel, we shall need the following lemma, which proof is an easy exercise and will be omitted.

Lemma 4.5 (Criterium for decrease). Let $\varphi:[0, T] \rightarrow \mathbb{R}$ be a continuous function, where $T \geq 0$. Assume that for every $0<\tau \leq T$, there exists $\delta>0$ such that for all $t \in(\tau-\delta, \tau) \cap[0, T]$ we have

Then $\varphi$ is decreasing.

$$
\varphi(t)>\varphi(\tau)
$$

We now prove that the generalized solutions of a convex foliation are self-contracted curves. Notice that in view of Theorem 3.3 this entails that these curves are rectifiable and have a finite length.

Theorem 4.6 (Self-contractedness of generalized orbits). Every convex foliation orbit is a (continuous) self-contracted curve.

Proof. Let $\gamma: I \rightarrow \mathbb{R}^{n}$ be a continuous injective curve which is a generalized solution of the convex foliation $\left\{C_{r}\right\}_{r \geq 0}$ in the sense of Definition 4.4 . Since $\gamma(I) \subset C_{r(0)}$, the curve is obviously bounded. Fix $\tau \in I$ and $\varepsilon>0$ such that $\tau+\varepsilon \in I$. We set

$$
\Gamma_{\varepsilon}:=\{\gamma(t): t \geq \tau+\varepsilon, t \in I\} .
$$

Notice that the compact set $\overline{\Gamma_{\varepsilon}}$ is contained in $\operatorname{int} C_{r\left(\tau+\varepsilon^{\prime}\right)}$ for all $0 \leq \varepsilon^{\prime}<\varepsilon$.

Claim 1. There exists $a>0$, such that for all $v \in N_{C_{r(\tau)}}(\gamma(\tau))$ and all $x \in \overline{\Gamma_{\varepsilon}}$ we have

$$
\left\langle v, \frac{x-\gamma(\tau)}{\|x-\gamma(\tau)\|}\right\rangle<-a<0 \text {. }
$$

Proof of Claim 1. Assume, towards a contradiction, that 4.6 fails. Since the sets $\overline{\Gamma_{\epsilon}}$ and $N_{C_{r(\tau)}}(\gamma(\tau)) \cap \mathbb{S}^{n-1}$ are compact, we easily deduce that for some unit normal $v \in N_{C_{r(\tau)}}(\gamma(\tau))$ and $x \in \overline{\Gamma_{\epsilon}}$ we have $\langle v, x-\gamma(\tau)\rangle \geq 0$. Since $x \in \operatorname{int} C_{r(\tau)}$, we deduce that for some $y \in C_{r(\tau)}$ we have $\langle v, y-\gamma(\tau)\rangle>0$. This contradicts the fact that $v \in N_{C_{r(\tau)}}(\gamma(\tau))$. Thus 4.6 holds.

Claim 2. For every $\varepsilon>0$ there exists $\delta>0$ such that for all $t^{-}, t^{+} \in I$ such that $t^{-} \in[\tau-\delta, \tau)$ and $t^{+} \geq \tau+\varepsilon$ we have

$$
\left\langle\gamma\left(t^{-}\right)-\gamma(\tau), \gamma\left(t^{+}\right)-\gamma(\tau)\right\rangle<0
$$

Proof of Claim 2. Fix $\varepsilon>0$ and let $a>0$ be given by Claim 1. Let $N^{1}=N_{C_{r(\tau)}}(\gamma(\tau)) \cap \mathbb{S}^{n-1}$ denote the (compact) set of unit normals at $\gamma(\tau)$, let $U_{\alpha}:=N^{1}+B(0, \alpha)$ be its $\alpha$-enlargement, and let $N_{\alpha}$ denote the closed convex cone generated by $U_{\alpha}$. It follows from 4.6 that if $\alpha>0$ is chosen small enough (depending on $a$ ), then for all $w \in N_{\alpha}$, and all $x \in \overline{\Gamma_{\epsilon}}$, we have $\langle w, x-\gamma(\tau)\rangle<0$. Since $\sec ^{-}(\tau) \subset N^{1} \subset U_{a}$, we deduce that for some $\delta>0$ we have

$$
\frac{\gamma(t)-\gamma(\tau)}{\|\gamma(t)-\gamma(\tau)\|} \in U_{\alpha}, \quad \text { for all } t \in(\tau-\delta, \tau) \cap I
$$

This establishes 4.7. 
Notice that (4.7) yields

$$
\left\|\gamma\left(t^{-}\right)-\gamma\left(t^{+}\right)\right\|>\left\|\gamma(\tau)-\gamma\left(t^{+}\right)\right\|
$$

We now prove that the curve $\gamma$ is self-contracted. Indeed, fix $0 \leq t_{1}<t_{2}<t_{3}$ in $I$ and consider the real-valued function

$$
\left\{\begin{array}{l}
\varphi:\left[0, t_{2}\right] \rightarrow \mathbb{R} \\
\varphi(t)=\left\|\gamma(t)-\gamma\left(t_{3}\right)\right\|
\end{array}\right.
$$

Applying Claim 2 for any $0<\tau \leq t_{2}$ and for $\varepsilon=t_{3}-t_{2}$ we deduce that for some $\delta_{1}>0$ and all $t \in\left(\tau-\delta_{1}, \tau\right) \cap\left[0, t_{2}\right]$ we have $\varphi(t)>\varphi(\tau)$. The conclusion follows from Lemma 4.5. Since $t_{1}, t_{2}, t_{3}$ are arbitrarily chosen we deduce that $\gamma$ is self-contracted.

As a consequence of Theorem 4.6 and [6, Proposition 2.2] the solution curve $\gamma$ has finite length. In particular, the curve converges as $t \rightarrow T_{\infty}$ to some limit point

$$
\gamma_{\infty}:=\lim _{t \rightarrow T_{\infty}} \gamma(t) \in \partial C_{r\left(T_{\infty}\right)}
$$

so that the mapping $\gamma$ can be continuously extended to $\left[0, T_{\infty}\right]$, by setting $\gamma\left(T_{\infty}\right):=\gamma_{\infty}$.

Remark 4.7 (Generalized versus classical solutions). Let $\gamma: I \rightarrow \mathbb{R}^{n}$ be a convex foliation orbit and $\Gamma=\gamma(I)$. Although $\gamma$ is merely continuous, Theorem 4.6 guarantees its rectifiability. Thus Proposition 3.4 applies, and the curve can be reparameterized by its arc-length to obtain a Lipschitz curve with the same image. The new curve $\gamma_{*}:[0, \ell(\gamma)] \rightarrow \mathbb{R}^{n}$ satisfies $\left\|\gamma_{*}^{\prime}(s)\right\|=1$ and

$$
-\gamma_{*}^{\prime}(s) \in N_{C(r(s))}\left(\gamma_{*}(s)\right)
$$

for almost all $s \in[0, \gamma(\ell)]$. It follows that every generalized orbit of a convex foliation gives rise to a classical solution (Lipschitz orbit).

4.1.3. Smooth solutions and strong self-contractedness. Let $\gamma: I \rightarrow \mathbb{R}^{n}$ be a continuous curve, where $I=\left[0, T_{\infty}\right)$, let $T \in I$ and denote by $\Omega(T)$ the convex hull of the tail of the curve, that is,

$$
\Omega(T):=\operatorname{conv}\{\gamma(t): t \geq T\} .
$$

Let us give the following definitions.

Definition 4.8 (Strong self-contractedness). A continuous injective curve $\gamma: I \rightarrow \mathbb{R}^{n}$ is called

- strictly self-contracted, if for $t_{1}, t_{2}, t_{3}$ in $I$ such that $t_{1}<t_{2}<t_{3}$ we have

$$
\left\|\gamma\left(t_{1}\right)-\gamma\left(t_{3}\right)\right\|>\left\|\gamma\left(t_{2}\right)-\gamma\left(t_{3}\right)\right\|
$$

- strongly self-contracted, if for all $\tau \in I$ we have

$$
\sec ^{-}(\tau) \subset \operatorname{int} N_{\Omega(\tau)}(\gamma(\tau))
$$

Of course every strictly self-contracted curve is self-contracted. A careful look at the proof of Theorem 4.6 actually reveals that the orbits of the convex foliation are strictly self-contracted curves. The following proposition relates the notions of strict and strong self-contractedness.

Proposition 4.9 (Strong versus strict self-contractedness). Every strongly self-contracted curve is strictly self-contracted. 
Proof. Fix $t_{1}<t_{2}<t_{3}$ in $I$ and apply 44.9$)$ for $\tau=t_{2}$. From the definition of $\sec ^{-}(\tau)$, see (4.3), we deduce that for some $\delta>0$ and all $t \in\left(t_{2}-\delta, t_{2}\right)$ we have

$$
\left\langle\gamma(t)-\gamma\left(t_{2}\right), \gamma\left(t_{3}\right)-\gamma\left(t_{2}\right)\right\rangle<0 .
$$

Setting $\varphi(t)=\left\|\gamma(t)-\gamma\left(t_{3}\right)\right\|$, the above inequality guarantees that Lemma 4.5 applies. The proof is complete.

Our next objective is to show that smooth orbits of a convex foliation are actually strongly self-contracted curves. We shall need the following lemma.

Lemma 4.10 (Differentiability point of a convex foliation orbit). Let $\gamma: I \rightarrow \mathbb{R}^{n}$ be a continuous orbit of a convex foliation and assume that $\gamma$ is differentiable at some $\tau>0$ with $\gamma^{\prime}(\tau) \neq 0$. Then

$$
-\gamma^{\prime}(\tau) \in \operatorname{int} N_{\Omega(\tau)}(\gamma(\tau))
$$

Proof. In order to simplify notation we assume $\gamma(\tau)=0$. (There is no loss of generality in doing this.) In view of Lemma 4.3 there exist $\varepsilon>0$, such that for all $t^{+} \in(\tau, \tau+\varepsilon)$ we have

$$
\left\langle\frac{\gamma^{\prime}(\tau)}{\left\|\gamma^{\prime}(\tau)\right\|}, \frac{\gamma\left(t^{+}\right)}{\left\|\gamma\left(t^{+}\right)\right\|}\right\rangle>\frac{1}{2}
$$

Since the compact set $\Omega(\tau+\varepsilon)$ is contained in int $C_{r(\tau)}$, arguing as in Claim 1 of the proof of Theorem 4.6, we deduce that for some $a>0$ and for all $t^{+} \geq \tau+\varepsilon$ we have

$$
\left\langle-\frac{\gamma^{\prime}(\tau)}{\left\|\gamma^{\prime}(\tau)\right\|}, \frac{\gamma\left(t^{+}\right)}{\left\|\gamma\left(t^{+}\right)\right\|}\right\rangle<-a .
$$

Changing $a$ into $\min (a, 1 / 2)$ if necessary, we deduce that 4.12 holds true for all $t^{+}>\tau$. We deduce that the tail of the curve for $t \geq \tau$ is contained in the convex cone

$$
\left\{x \in \mathbb{R}^{n}:\left\langle\frac{\gamma^{\prime}(\tau)}{\left\|\gamma^{\prime}(\tau)\right\|}, x\right\rangle \leq-a\|x\|\right\},
$$

hence so does $\Omega(\tau)$. This easily yields $-\gamma^{\prime}(\tau) \in \operatorname{int} N_{\Omega(\tau)}(\gamma(\tau))$.

Corollary 4.11 (Strong self-contractedness of smooth orbits). Every $C^{1}$ convex foliation orbit with no stationary point is a strongly self-contracted curve.

Proof. Parameterizing the curve by its arc-length parametrization we obtain a $C^{1}$ curve with nonvanishing derivative and the same image $\Gamma=\gamma(I)$. This new curve satisfies $\|\dot{\gamma}(s)\|=1$, for every $s \in[0, \ell(\gamma)]$ and it is also a convex foliation orbit, see Remark 4.7. The result follows by combining Lemma 4.3 with Lemma 4.10.

4.2. Polygonal approximations of smooth strongly self-contracted curves. In this section we prove that every strongly self-contracted $C^{1}$ curve is a limit of self-contracted polygonal curves (with respect to the Hausdorff distance). Let us recall the relevant definition.

Definition 4.12 (Polygonal approximation). Let $\gamma: I \rightarrow \mathbb{R}^{n}$ be a continuous curve. A polygonal line $P=\bigcup_{k=0}^{m}\left[z_{k+1}, z_{k}\right]$ is called polygonal approximation of accuracy $\delta>0$ for the curve $\gamma$, if

$$
\left\{z_{k}\right\}_{k} \subset \gamma(I), \quad z_{k+1} \preceq z_{k} \quad \text { and } \quad d_{H}(P, \gamma(I)) \leq \delta .
$$


In the above we used the same notation $x \preceq y$ which corresponds to the order on the curve as in the beginning of the proof of Theorem 3.3. In the sequel, bounded continuous self-contracted curves $\gamma: I \rightarrow \mathbb{R}^{n}$ are considered to be extended to $\bar{I}$, so that $\Gamma=\gamma(\bar{I})$ is a compact set.

Proposition 4.13 (Self-contracted polygonal approximations). Let $\gamma:[0, L] \rightarrow \mathbb{R}^{n}$ be a $C^{1}$ strongly self-contracted curve. Then for every $\delta>0$ the curve $\gamma$ admits a self-contracted polygonal approximation of accuracy $\delta>0$.

Proof. Since the statement is independent of the parametrization, there is no loss of generality in assuming that the curve is parameterized by arc-length. In particular, $\left\|\gamma^{\prime}(t)\right\|=1$ for all $t \in[0, L]$ where $L$ is the total length of the curve. Moreover, 4.10 holds.

We shall show that for every $\delta>0$ and every $T_{0} \in(0, L]$ there exists a polygonal approximation $P_{\delta}\left(T_{0}\right)=\bigcup_{k=0}^{m}\left[z_{k+1}, z_{k}\right]$ of the curve $\gamma\left(\left[T_{0}, L\right]\right)$ of accuracy $\delta>0$. Indeed, let $T_{0} \in(0, L]$ and set $z_{0}=\gamma\left(T_{0}\right)$. In view of 4.10 and Lemma 4.3 there exists $t^{-} \in\left[T_{0}-\delta, T_{0}\right)$ such that

$$
\frac{\gamma\left(t^{-}\right)-\gamma\left(T_{0}\right)}{\left\|\gamma\left(t^{-}\right)-\gamma\left(T_{0}\right)\right\|} \in \operatorname{int} N_{\Omega\left(T_{0}\right)}\left(\gamma\left(T_{0}\right)\right)
$$

Let $T_{1}>0$ be the infimum of all $t^{-} \in\left[T_{0}-\delta, T_{0}\right)$ such that 4.13 holds, and set $z_{1}=\gamma\left(T_{1}\right)$. Notice that $\left(z_{1}-z_{0}\right) \in N_{\Omega\left(T_{0}\right)}\left(\gamma\left(T_{0}\right)\right)$, which yields that for any $x \in \Omega\left(T_{0}\right)$, the function

$$
t \mapsto d\left(x, z_{0}+t\left(z_{1}-z_{0}\right)\right)
$$

is increasing. Thus the curve $\left[z_{1}, z_{0}\right] \cup\left\{\gamma(t): t \geq T_{0}\right\}$ is self-contracted. Since $t \mapsto \gamma(t)$ is the length parametrization, it follows that $\left\|z_{1}-z_{0}\right\| \leq\left|T_{1}-T_{0}\right| \leq \delta$ whence

$$
d_{H}\left(\left[z_{1}, z_{0}\right], \gamma\left(\left[T_{1}, T_{0}\right]\right)\right) \leq \delta .
$$

Notice finally that

$$
\left[z_{1}, z_{0}\right] \cup \Omega\left(T_{0}\right) \subset \Omega\left(T_{1}\right) .
$$

Repeating the above procedure, we build after $m$ iterations, a decreasing sequence $T_{0}>T_{1}>$ $\ldots>T_{m+1}$ and points $z_{k}=\gamma\left(T_{k}\right)$, such that $\bigcup_{k=0}^{m}\left[z_{k+1}, z_{k}\right]$ is a self-contracted polygonal approximation of the curve $\left\{\gamma(t): t \in\left[T_{m+1}, T_{0}\right]\right\}$ of precision $\delta>0$. Notice also that $\Omega\left(T_{0}\right) \cup \bigcup_{k=0}^{m}\left[z_{k+1}, z_{k}\right] \subset \Omega\left(T_{m+1}\right)$.

The proof will be complete if we show that with this procedure we reach the initial point $t=0$ in a finite number of iterations, i.e. $T_{m+1}=0$ for some $m \geq 1$. Let us assume this is not the case. Then the aforementioned procedure gives a decreasing sequence $\left\{T_{m}\right\}_{m} \searrow T$, where $T \geq 0$. Set $z=\gamma(T)$ and $z_{m}=\gamma\left(T_{m}\right)$ and notice that $\left\{z_{m}\right\} \rightarrow z$. We may assume that $\left\{z_{m}\right\}_{m \geq m_{0}} \subset B(z, \delta)$. It follows that for every $m \geq m_{0}$ there exists $s_{m}>0$ and $x_{m}:=\gamma\left(T_{m}+s_{m}\right)$ such that

$$
\left\langle\frac{z-z_{m}}{\left\|z-z_{m}\right\|}, \frac{x_{m}-z_{m}}{\left\|x_{m}-z_{m}\right\|}\right\rangle>0 \text {. }
$$

(If (4.14) were not true, then we could have taken $z_{m+1}=z$ and $T_{m+1}=T$ a contradiction to the definition of $T_{m+1}$ as an infimum.) Let us now assume that a subsequence of $\left\{x_{m}\right\}_{m}$ remains away of $z$. Then taking a converging sub-subsequence $\left\{z_{k_{m}}\right\}_{m}$ and passing to the limit in 4.14 as $m \rightarrow \infty$, we obtain that $\left\langle\gamma^{\prime}(T), u\right\rangle \geq 0$, for some unit vector $u$ in the cone over $\Omega(T)-z$, which contradicts 4.9 . It follows that $s_{m} \rightarrow 0$ and $\left\{x_{m}\right\}_{m} \rightarrow z$. 
For the rest of the proof, let us assume for simplicity that $z=0$ and $\gamma^{\prime}(T)=e_{n}=(0, \ldots, 1)$. (There is no loss of generality in doing so.) Since $\gamma$ is a $C^{1}$ curve, it follows that for any $\varepsilon>0$, there exists $\tau>0$ such that for all $t \in(T, T+\tau)$ we have

$$
1 \geq\left\langle e_{n}, \gamma^{\prime}(t)\right\rangle \geq 1-\varepsilon
$$

and

$$
\left\|e_{n}-\frac{\gamma(t)}{\|\gamma(t)\|}\right\| \leq \varepsilon
$$

Pick any $\bar{m}$ sufficiently large so that $T_{\bar{m}}+s_{\bar{m}}<\tau$, set $\bar{z}=z_{\bar{m}}=\gamma\left(T_{\bar{m}}\right)$ and $\bar{x}=x_{\bar{m}}=\gamma\left(T_{\bar{m}}+s_{\bar{m}}\right)$. Since $\bar{x}, \bar{z}$ satisfy (4.14) and (4.16) we deduce that

$$
\left\langle\frac{\bar{z}}{\|\bar{z}\|}, \frac{\bar{x}-\bar{z}}{\|\bar{x}-\bar{z}\|}\right\rangle<0 \quad \text { and } \quad\left\|e_{n}-\frac{\bar{z}}{\|\bar{z}\|}\right\|<\varepsilon
$$

hence $\left\langle e_{n}, \frac{\bar{x}-\bar{z}}{\|\bar{x}-\bar{z}\|}\right\rangle<\varepsilon$, and the last coordinates satisfy

$$
\bar{x}^{n}-\bar{z}^{n}<\varepsilon\|\bar{x}-\bar{z}\|=\varepsilon\left\|\gamma\left(T_{\bar{m}}+s_{\bar{m}}\right)-\gamma\left(T_{\bar{m}}\right)\right\| \leq \varepsilon s_{\bar{m}} .
$$

On the other hand, using (4.15) we deduce

$$
\bar{x}^{n}-\bar{z}^{n}=\int_{0}^{s_{\bar{m}}}\left\langle e_{n}, \operatorname{gamma}^{\prime}\left(T_{\bar{m}}+s\right)\right\rangle d s \geq(1-\varepsilon) s_{\bar{m}} .
$$

Relations (4.17), 4.18) are incompatible for $\varepsilon<1 / 2$, leading to a contradiction.

This proves that for some $m$ we get $T_{m+1}=0$ and $z_{m+1}=\gamma(0)$. The proof is complete.

In view of Corollary 4.11 we thus obtain.

Corollary 4.14. Every $C^{1}$ convex foliation orbit admits self-contracted polygonal approximations of arbitrary accuracy.

4.3. Convergence of the proximal algorithm. The aim of this section is to provide a different type of application of the notion of self-contracted curves. We recall that for a (nonsmooth) convex function $f: \mathbb{R}^{n} \rightarrow \mathbb{R}$ which is bounded from below, an initial point $x_{0} \in \mathbb{R}^{n}$ and a sequence $\left\{t_{i}\right\}_{i} \subset(0,1]$, the algorithm defines a sequence $\left\{x_{i}\right\}_{i \geq 0} \subset \mathbb{R}^{n}$ called proximal sequence according to the iteration scheme, see [16, Definition 1.22] for example:

Given $x_{i}$, define $x_{i+1}$ as the (unique) solution of the following (strongly convex, coercive) minimization problem

$$
\min _{x}\left\{f(x)+\frac{1}{2 t_{i}}\left\|x-x_{i}\right\|^{2}\right\}
$$

A necessary and sufficient optimality condition for the above problem is

$$
0 \in \partial f\left(x_{i+1}\right)+t_{i}^{-1}\left(x_{i+1}-x_{i}\right)
$$

or equivalently,

$$
\frac{x_{i+1}-x_{i}}{t_{i}} \in-\partial f\left(x_{i+1}\right),
$$

where $\partial f$ is the Fenchel subdifferential of $f$. Notice that 4.20 can be seen as an implicit discretization of the subgradient system $(1.2)$ or of the gradient system (1.1), in case $f$ is smooth.

The proximal algorithm has been introduced in [14]. We refer to [10] for a geometrical interpretation and to [4] for extensions to nonconvex problems. Let us now recall from [1, Section 2.4] the following important facts (we give the short proof for completeness): 
Lemma 4.15 (Geometrical interpretation). Let $\left\{x_{i}\right\}_{i \geq 0}$ be a proximal sequence for a convex function $f$ and set $r_{i}:=f\left(x_{i}\right)$. Then:

(i) $f\left(x_{i+1}\right)<f\left(x_{i}\right)$ if $x_{i+1} \neq x_{i}$;

(ii) the point $x_{i+1}$ is the shortest distance projection of $x_{i}$ on the sublevel set $\left[f \leq r_{i+1}\right]$.

Proof. Assertion (i) is obvious, since $x_{i+1}$ minimizes 4.19), thus for $x=x_{i}$ we get

$$
f\left(x_{i+1}\right)+\frac{1}{2 t_{i}}\left\|x_{i+1}-x_{i}\right\|^{2} \leq f\left(x_{i}\right) .
$$

Assertion (ii) follows again from (4.19), by considering $x \in\left[f \leq r_{i+1}\right]$. Indeed:

$$
f\left(x_{i+1}\right)+\frac{1}{2 t_{i}}\left\|x_{i+1}-x_{i}\right\|^{2} \leq f(x)+\frac{1}{2 t_{i}}\left\|x-x_{i}\right\|^{2} \leq r_{i+1}+\frac{1}{2 t_{i}}\left\|x-x_{i}\right\|^{2} .
$$

Since $r_{i+1}=f\left(x_{i+1}\right)$ the above yields

$$
\left\|x_{i+1}-x_{i}\right\| \leq\left\|x-x_{i}\right\|, \text { for all } x \in\left[f \leq r_{i+1}\right] .
$$

The proof is complete.

We obtain the following interesting consequence.

Proposition 4.16 (Self-contractedness of the proximal algorithm). Let $\left\{x_{i}\right\}_{i \geq 0}$ be a proximal sequence for a convex function $f$. Then the polygonal curve

$$
P=\bigcup_{i \in \mathbb{N}}\left[x_{i}, x_{i+1}\right]
$$

is a self-contracted curve.

Proof. Let $i_{1}<i_{2}<i_{3}$. Notice first that for every $x \in\left[f \leq r_{m+1}\right]$, the function

$$
t \mapsto\left\|x_{m+1}+t\left(x_{m}-x_{m+1}\right)-x\right\|
$$

is increasing because the sublevel sets are convex. Now Lemma 4.15 yields that for all $i_{1} \leq m<$ $i_{2}, x_{i_{3}} \in\left[f \leq f\left(x_{m+1}\right)\right]$ and

$$
\left\|x_{m+1}-x_{i_{3}}\right\| \leq\left\|x_{m}-x_{i_{3}}\right\|
$$

which inductively yields

$$
\left\|x_{i_{2}}-x_{i_{3}}\right\| \leq\left\|x_{i_{1}}-x_{i_{3}}\right\| \text {. }
$$

A simple argument now shows that the polygonal curve is self-contracted.

Combining the above with the length bound given in (2.3) (in view of Remark 3.5) we obtain the following convergence result.

Theorem 4.17 (Convergence of the proximal algorithm). Let $f: \mathbb{R}^{n} \rightarrow \mathbb{R}$ be convex and bounded from below. Let $x_{0} \in \mathbb{R}^{n}$ be an initial point for the proximal algorithm with parameters $\left\{t_{i}\right\}_{i} \subset(0,1]$. Then the proximal sequence $\left\{x_{i}\right\}_{i}$ converges to some point $x_{\infty} \in \mathbb{R}^{n}$ with a fast rate of convergence. That is, there exists $c>0$, depending only on the dimension, such that

$$
\sum_{i \in \mathbb{N}}\left\|x_{i}-x_{i+1}\right\| \leq c\left\|x_{0}-x_{\infty}\right\| .
$$


Proof. By Proposition 4.16 the polygonal curve $P$ defined in 4.21 is self-contracted. Consequently, by Remark 3.5 we know that (2.3) applies, yielding

$$
\ell(P)=\sum_{n \in \mathbb{N}}\left\|x_{i}-x_{i+1}\right\| \leq C_{n} \operatorname{diam} K,
$$

where $K$ is any compact convex set containing $\left\{x_{i}\right\}_{i}$. In addition, letting $i_{1}=0$ and $i_{3} \rightarrow+\infty$ in 4.22 we get

$$
\left\|x_{i}-x_{\infty}\right\| \leq\left\|x_{0}-x_{\infty}\right\|,
$$

for all $i \in \mathbb{N}$, which implies that $\left\{x_{i}\right\}_{i} \subset \bar{B}\left(x_{\infty}, R\right)$ with $R=\left\|x_{0}-x_{\infty}\right\|$ and the result follows for $c=2 C_{n}$.

Theorem 4.17 guarantees the fast convergence of the proximal sequence $\left\{x_{i}\right\}_{i}$ towards a limit point, with a convergence rate independent of the choice of the parameters $\left\{t_{i}\right\}_{i}$. Notice however that for an arbitrary choice of parameters, the limit point might not be a critical point of $f$ as shows the following example:

Example 4.18 (Convergence to a noncritical point). Consider the (coercive, $C^{1}$ ) convex function $f: \mathbb{R} \rightarrow \mathbb{R}$ defined by

$$
f(x)=\left\{\begin{array}{cc}
x^{2}, & |x| \leq 1 / 2 \\
|x|-\frac{1}{4}, & |x| \geq 1 / 2
\end{array}\right.
$$

Then the proximal algorithm $\left\{x_{i}\right\}_{i}$, initialized at the point $x_{0}=2$ and corresponding to the parameters $t_{i}=1 / 2^{i+1}, i \in \mathbb{N}$, converges to the noncritical point $\bar{x}=1$.

In the above example, the choice of the parameters $\left\{t_{i}\right\}$ in the proximal algorithm has the drawback that $\sum_{i} t_{i}<+\infty$. In practice the choice of the step-size parameters $\left\{t_{i}\right\}_{i}$ is crucial to obtain the convergence of the sequence $\left\{f\left(x_{i}\right)\right\}_{i}$ towards a critical value; a standard choice for this is any sequence satisfying $\sum t_{i}=+\infty$, see for instance [8].

\section{REFERENCES}

[1] Bolte, J., Danillidis, A., Ley, O., Mazet, L., Characterizations of Łojasiewicz inequalities: subgradient flows, talweg, convexity, Trans. Amer. Math. Soc. 362 (2010), 3319-3363.

[2] BrÉzIs, H., Opérateurs maximaux monotones et semi-groupes de contractions dans les espaces de Hilbert (French), North-Holland Mathematics Studies 5, (North-Holland Publishing Co., 1973).

[3] Burago, D., Burago, Y., Ivanov, S., A course in metric geometry, Graduate Studies in Mathematics, Vol. 33, American Mathematical Society, Providence, RI, 2001.

[4] Combettes, P. \& Pennanen, T., Proximal methods for cohypomonotone operators, SIAM J. Control Optim. 43 (2004), 731-742.

[5] Danillidis, A., Garcia Ramos, Y., Some remarks on the class of continuous (semi-)strictly quasiconvex functions, J. Optim. Theory Appl. 133 (2007), 37-48.

[6] Danillidis, A. Ley, O., Sabourau, S., Asymptotic behaviour of self-contracted planar curves and gradient orbits of convex functions, J. Math. Pures Appl. 94 (2010), 183-199.

[7] Giannotti, C. Spiro, A., Steepest descent curves of convex functions on surfaces of constant curvature, Isr. J. Math. 191 (2012), 279-306.

[8] GülER, O. On the convergence of the proximal point algorithm for convex minimization, SIAM J. Control Optim. 29 (1991), 403-419.

[9] Kurdyka, K., On gradients of functions definable in o-minimal structures, Ann. Inst. Fourier 48 (1998), 769-783.

[10] Lemaire, B. About the convergence of the proximal method. Advances in optimization (Lambrecht, 1991), 3951, Lecture Notes in Econom. and Math. Systems 382, Springer, Berlin, 1992.

[11] Łojasiewicz, S., "Une propriété topologique des sous-ensembles analytiques réels.", in: Les Équations aux Dérivées Partielles, pp. 87-89, Éditions du centre National de la Recherche Scientifique, Paris, 1963. 
[12] Manselli, P. PuCci, C., Uniqueness results for evolutes and self-evolvents, Boll. Un. Mat. Ital. A 5 (1991), $373-379$.

[13] Manselli, P. Pucci, C., Maximum length of steepest descent curves for quasi-convex functions, Geom. Dedicata 38 (1991), 211-227.

[14] Martinet, B., Régularisation d'inéquations variationnelles par approximations successives. (French) Rev. Française Informat. Recherche Opérationnelle, 4, Ser. R3 (1970) 154-158.

[15] Palis, J. \& De Melo, W., Geometric theory of dynamical systems. An introduction, (Translated from the Portuguese by A. K. Manning), Springer-Verlag, New York-Berlin, 1982.

[16] Rockafellar, R.T. \& Wets, R., Variational Analysis, Grundlehren der Mathematischen, Wissenschaften, Vol. 317, (Springer, 1998).

Aris Daniilidis

Departament de Matemàtiques, $\mathrm{C} 1 / 308$

Universitat Autònoma de Barcelona

E-08193 Bellaterra, Spain

E-mail: arisd@mat.uab.es

http://mat.uab.es/ arisd

Research supported by the grant MTM2011-29064-C01 (Spain).

Guy David

Laboratoire de mathématiques, UMR 8628, Bâtiment 425

Université Paris-Sud, F-91405 Orsay Cedex, France

et Institut Universitaire de France

E-mail: Guy.David@math.u-psud.fr

http://www.math.u-psud.fr/〜gdavid/

Estibalitz Durand-Cartagena

Departamento de Matemática Aplicada

ETSI Industriales, UNED

Juan del Rosal 12, Ciudad Universitaria, E-28040 Madrid, Spain

E-mail: edurand@ind.uned.es

https://dl.dropbox.com/u/20498057/Esti/Home_Page.html

Antoine Lemenant

Laboratoire Jacques Louis Lions (CNRS UMR 7598)

Université Paris 7 (Denis Diderot)

175, rue du Chevaleret, F-75013 Paris, France

E-mail: lemenant@ljll.univ-paris-diderot.fr

http://www.ann.jussieu.fr/ Iemenant/ 